\title{
A FIXED POINT THEOREM WITH APPLICATIONS TO CONVOLUTION EQUATIONS
}

\author{
R. E. EDWARDS \\ (received 26 November 1962)
}

\section{Introduction and summary}

The well-known Banach Contraction Principle asserts that any self-map $F$ of a complete metric space $M$ with the property that, for some number $k<1$,

$$
d(F(x), F(y)) \leqq k \cdot d(x, y)
$$

for all $x, y \in M$, possesses a unique fixed point in $M$. Some extensions and analogues have recently been given by Edelstein [1]. For the reader's convenience we state here the result of Edelstein which we shall employ. It asserts that if $F$ is a self-map of a metric space $M$ having the property that

$$
d(F(x), F(y))<d(x, y)
$$

for any two distinct points $x$ and $y$ of $M$, and if $x_{0}$ is a point of $M$ such that the sequence of iterates $x_{n}=F^{n}\left(x_{0}\right)$ contains a subsequence which converges in $M$, then the limit of this subsequence is the unique fixed point of $F$.

Our aim is to apply these results to the case in which $M$ is a subset of some topological vector space, the metric $d$ being chosen in a manner dependent in some measure upon $F$. The resulting general theorems have applications to the study of convolution equations over groups, yielding results more refined than those stemming from the Contraction Principle applied to the initial metric on the spaces involved.

\section{Notations and definitions}

In the general theorems to follow, $E$ will denote a topological vector space, $M$ a subset of $E$, and $F$ a self-map of $M$. It is evident that the following condition, (A), is necessary for the existence of a fixed point of $F$ :

(A) There exists a point $x_{0}$ of $M$ such that the iterates

$$
x_{n}=F^{n}\left(x_{0}\right) \quad(n=1,2, \cdots)
$$

for a bounded subset of $E$. 
The aim is to combine (A) with a type of contraction hypothesis on $F$ in such a way as to obtain sufficient conditions in order that $F$ shall admit at least one fixed point.

Although we do not assume outright that $E$ is locally convex, yet we shall introduce the weakened topology $\sigma\left(E, E^{\prime}\right)$ on $E, E^{\prime}$ denoting the topological dual of $E$; this topology may be the zero topology (having $\phi$ and $E$ as the only open sets). On $E^{\prime}$ we shall employ the strong topology $\beta\left(E^{\prime}, E\right)$, that of convergence uniform on the bounded subsets of $E$.

The contraction hypothesis on $F$ will be framed in terms of a family of seminorms on $E$, concerning which we introduce a definition.

Definition. By an adequate family of seminorms on $E$ is meant a countable family $\left(p_{m}\right)$ such that

(i) each $p_{m}$ is a weakly continuous seminorm on $E$;

(ii) the family $\left(p_{m}\right)$ is strongly bounded, i.e.,

$$
\sup _{m} p_{m}(B)<+\infty
$$

for each bounded subset $B$ of $E$;

(iii) the vector subspace $V$ of $E^{\prime}$, generated by those linear forms on $E$ which are continuous (i.e., bounded) with respect to some $p_{m}$, is strongly dense in $E^{\prime}$.

If condition (i) is weakened to

(i') each $p_{m}$ is a seminorm on $E$ which is sequentially weakly continuous, i.e., $\lim _{n} p_{m}\left(x_{m}\right)=0$ for each sequence $\left(x_{n}\right)$ converging weakly to 0 in $E$, then we speak of $\left(p_{m}\right)$ as a 'adequate family.

Remarks. (1) If $E$ is an infrabarrelled space, (ii) is satisfied whenever the family $\left(p_{m}\right)$ is bounded at each point of $E$, i.e., whenever

$$
\sup _{m} p_{m}(x)<+\infty
$$

for each $x$ in $E$.

(2) If $E$ is separated and locally convex, condition (ii) entails that the $p_{m}$ separate points of $E$.

(3) There is a real distinction between (i) and $\left(i^{\prime}\right)$. On the one hand, a seminorm $p$ on $E$ is weakly continuous if and only if there is a finite family $\left(x_{q}^{\prime}\right)_{1 \leqq q \leqq r}$ of points of $E^{\prime}$ such that

$$
p(x) \leqq \sum_{q=1}^{\prime}\left|\left\langle x, x_{q}^{\prime}\right\rangle\right|
$$

for all $x$ in $E$. On the other hand, a seminorm $p$ on $E$ is sequentially weakly continuous whenever it satisfies an inequality

$$
p(x) \leqq \int_{Q}\left|\left\langle x, x^{\prime}(q)\right\rangle\right| d \mu(q)
$$


for all $x$ in $E, Q$ being a separated locally compact space, $\mu$ a positive Radon measure on $Q$, and $q \rightarrow x^{\prime}(q)$ being an $E^{\prime}$-valued function on $Q$ which is such that the scalar-valued function $q \rightarrow\left\langle x, x^{\prime}(q)\right\rangle$ is $\mu$-measurable for each $x$ in $E$, and such that furthermore

$$
\int_{Q}^{*} \sup _{x \in B}\left|\left\langle x, x^{\prime}(q)\right\rangle\right| d \mu(q)<+\infty
$$

for each bounded subset $B$ of $E$. That weak sequential continuity obtains under these conditions, is a simple consequence of the Lebesgue dominated convergence theorem.

For example, if $T$ is a separated compact space and $E$ the Banach space of real - or complex - valued continuous functions on $T$ (with the usual maximum modulus norm), consider the seminorm

$$
p(x)=\int_{T}|x(t)| d \mu(t),
$$

$\mu$ being a positive Radon measure on $T$. Then $p$ is sequentially weakly continuous, whatever the choice of $\mu$; but it is weakly continuous if and only if the support of $\mu$ is finite.

\section{Statement of the main theorems}

These are two in number.

TheOREM 1. Let $E$ be a topological vector space, $M$ a subset of $E$, and $F$ a self-map of $M$. Suppose that $M$ is sequentially weakly complete, that (A) holds, and that furthermore the following condition, (B), is fulfilled:

(B) There exists a'-adequate family $\left(p_{m}\right)$ and a number $k<1$ such that

$$
p_{m}(F(x)-F(y)) \leqq k \cdot p_{m}(x-y)
$$

for all $m$ and all $x, y \in M$.

Then the sequence $\left(x_{n}\right)=\left(F^{n}\left(x_{0}\right)\right)$ converges weakly in $E$ to a point $x_{\infty} \in M$ such that

$$
p_{m}\left(F\left(x_{\infty}\right)-x_{\infty}\right)=0
$$

for all $m$. If furthermore the $p_{m}$ separate the points of $E, x_{\infty}$ is the unique fixed point of $F$.

THEOREM 2. Let $E$ be a topological vector space, $M$ a subset of $E, F$ a self-map of $M$. Suppose that the following two conditions, $\left(\mathrm{A}^{\prime}\right)$ and $\left(\mathrm{B}^{\prime}\right)$, are fulfilled:

( $\left.\mathrm{A}^{\prime}\right)$ There exists a point $x_{0}$ of $M$ such that the sequence $\left(x_{n}\right)=\left(F^{n}\left(x_{0}\right)\right)$ has a bounded subsequence admitting a weak limiting point $x_{\infty} \in M$.

(B') There exists an adequate family $\left(p_{m}\right)$ such that

$$
p_{m}(F(x)-F(y)) \leqq p_{m}(x-y)
$$


for all $m$ and all $x, y \in M$; and if $x$ and $y$ are distinct points of $M$, there exists an index $m$ such that

$$
p_{m}(F(x)-F(y))<p_{m}(x-y) .
$$

Then $x_{\infty}$ is the unique fixed point of $F$.

Finally, if in ( $\left.\mathrm{A}^{\prime}\right)$ it be assumed that $\left(x_{n}\right)$ admits a subsequence converging weakly to a point $x_{\infty} \in M$, then in $\left(\mathrm{B}^{\prime}\right)$ it suffices to assume that the family $\left(p_{m}\right)$ is '-adequate.

Remark. In the same vein as the final statement of Theorem 2 it may be noted that if in $\left(\mathrm{A}^{\prime}\right)$ it be assumed that a bounded subsequence of $\left(x_{n}\right)$ exists which admits a limiting point $x_{\infty} \in M$, then weak continuity of each $p_{m}$ may be relaxed to continuity.

\section{Proofs of the main theorems}

It will suffice to deal with Theorem 2, which is the more interesting result. The same general technique, together with a few minor variations, suffices to establish Theorem 1 .

Introduce into $E$ the metric

$$
d(x, y)=\sum_{m} m^{-2} p_{m}(x-y),
$$

it being supposed that the family $\left(p_{m}\right)$ is indexed by some subset of the set of natural numbers. The second clause of $\left(\mathrm{B}^{\prime}\right)$ ensures that $d(x, y)>0$ if $x \neq y$.

Suppose that $\left(x_{n_{k}}\right)$ is a bounded subsequence of $\left(x_{n}\right)$ admitting a weak limiting point $x_{\infty} \in M$. On each bounded subset $B$ of $E$, hence in particular on the bounded subset $\left\{x_{n_{k}}\right\} \cup\left\{x_{\infty}\right\}$, the weakened topology induces a topology stronger than that defined by $d$. This is apparent from the inequality

$$
\left|d(x, y)-\sum_{m \leqq r} m^{-2} p_{m}(x-y)\right| \leqq 2 c \cdot \sum_{m>r} m^{-2},
$$

valid for all $x, y \in B$ and $c=\sup _{m} p_{m}(B)<+\infty$, combined with the assumed weak continuity of each $p_{m}$. It therefore follows that $x_{\infty}$ is a limiting point of $\left(x_{n_{k}}\right)$ for the topology defined by $d$, so that a subsequence $\left(x_{n}^{*}\right)$ of $\left(x_{n}\right)$ exists for which $d\left(x_{n}^{*}, x_{\infty}\right) \rightarrow 0$ as $n \rightarrow \infty$.

Besides this, if $x$ and $y$ are distinct points of $\bar{M},\left(\mathrm{~B}^{\top}\right)$ entails that

$$
d(F(x), F(y))<d(x, y) .
$$

At this stage we may appeal to Edelstein's theorem to conclude that $x_{\infty}$ is the unique fixed point of $F$.

Finally, if in $\left(\mathrm{A}^{\prime}\right)$ we assume that $\left(x_{n}\right)$ has a subsequence $\left(x_{n}^{*}\right)$ converging weakly to $x_{\infty} \in M$, and if $\left(p_{m}\right)$ is '-adequate, we may still infer that 
$d\left(x_{n}^{*}, x_{\infty}\right) \rightarrow 0$ as $n \rightarrow \infty$. It suffices for this to use (1) once again, taking $x=x_{n}^{*}$ and $y=x_{\infty}$, and use the weak sequential continuity of each $p_{m}$. Edelstein's theorem can then be applied as before.

\section{Supplements to theorems 1 and 2}

In either theorem it is admissible to replace the weakened topology $\sigma\left(E, E^{\prime}\right)$ by a weak topology $\sigma(E, L)$, where $L$ is some subspace of $E^{\prime}$, provided that at the same time one demands that (a) weak sequential continuity of $p_{m}$ be replaced by sequential continuity relative to the topology $\sigma(E, L)$, and (b) the vector subspace $V$ figuring in condition (iii) of $\S 1$ is assumed to have a strong closure containing $L$.

This remark is useful in case $E$ is itself the dual, $X^{\prime}$, of some topological vector space $X$, in which case one might wish to take for $L$ the image of $X$ under the natural injection of $X$ into $X^{\prime \prime}$. If $X$ is infrabarrelled, one would then require that $V$ be a dense vector subspace of $X$. For more general spaces $X$, the strong topology on $X^{\prime \prime}$ will in general induce on $X$ a topology stronger than the initial one.

\section{Affine linear maps}

Consider the case in which $F$ takes the form

$$
F(x)=A x+y \text {, }
$$

where $y \in E$ is given and $A$ is a continuous endomorphism of $E$. An application of Theorem 2 leads to the following result.

THEOREM 3. Let $E$ be a topological vector space, $A$ a continuous endomorphism of $E$, and $A^{\prime}$ the adjoint of $A$ (an endomorphism of $E^{\prime}$ ). Suppose that $A^{\prime}$ has a countable, strongly bounded family $\left(e_{m}^{\prime}\right)$ of eigenvectors, each belonging to an eigenvalue of modulus inferior to 1 , which are total over $E$ and which generate a strongly dense vector subspace of $E^{\prime}$. Let $y \in E$ be given. If there exists $x \in E$ such that the sequence $\left(x_{n}\right)$ defined by

$$
x_{n}=A^{n} x_{0}+y+A y+\cdots+A^{n-1} y
$$

admits a bounded subsequence admitting a weak limiting point $x_{\infty} \in E$, then the equation

$$
x-A x=y
$$

has $x=x_{\infty}$ as its unique solution in $E$.

Proof. Suppose that $A^{\prime} e_{m}^{\prime}=\lambda_{m} e_{m}^{\prime}$, where $\left|\lambda_{m}\right|<1$. Put $F(x)=A x+y$ and $p_{m}(x)=\left|\left\langle x, e_{m}^{\prime}\right\rangle\right|$ for $x \in E$. The hypotheses of Theorem 2 are then fulfilled, and an application of that theorem leads to the stated conclusion.

Naturally, Theorem 1 leads to a corresponding result. 


\section{Convolution equations over compact groups}

In this section the aim is to apply Theorems 2 and 3 , supplemented where necessary by the remarks in $\$ 4$, to the study of convolution equations of the type

$$
x-\mu * x=y,
$$

$\mu$ being a Radon measure, and $x$ and $y$ Radon measures or functions, on a compact group $T$. We adopt the usual notation, $L^{p}(T)$, for the various Lebesgue spaces formed with respect to Haar measure on $T$. In addition, $M(T)$ will denote the space of all Radon measures on $T$, normed in the usual way as the dual of the Banach space $C(T)$ of continuous, complex-valued functions on $T$. The symbol $\{U\}$ is used to denote a complete set of inequivalent, irreducible, continuous, unitary representations of $T$. If $x$ belongs to $L^{p}(T)$ [or to $M(T)$ ] we write

$$
\hat{x}(U)=\int_{T} x(t) U(t) d t \quad\left[\text { or } \int_{r} U(t) d x(t)\right]
$$

for each $U \in\{U\}$. If $H(U)$ is the representation space corresponding to $U$ (a finite-dimensional Hilbert space), each $U(t)$ is a unitary endomorphism of $H(U)$ and $\hat{x}(U)$ is an endomorphism of $H(U)$ too. $\|\hat{x}(U)\|$ denotes the usual norm of the endomorphism $\hat{x}(U)$.

Our arguments make use of the consequences of the Peter-Weyl theory in so far as they affect harmonic analysis, amongst which we note particularly the Parseval formula

$$
\|x\|_{L^{2}}^{2}=\sum d(U) \cdot \operatorname{Tr}\left[\hat{x}(U)^{*} \hat{x}(U)\right]
$$

for $x \in L^{2}(T)$; here the sum extends over $\{U\}, d(U)$ is the dimension or degree of the representation $U$, "Tr" is short for "trace", and $\hat{x}(U)^{*}$ is the Hilbert adjoint of $\hat{x}(U)$. Since also

$$
\begin{aligned}
& \|A\| \leqq\left(\operatorname{Tr} A^{*} A\right)^{\underline{t}}, \\
& \|\hat{x}(U)\| \leqq\|x\|_{L^{2}},
\end{aligned}
$$

and $L^{2}(T)$ is dense in $L^{1}(T)$, it follows that

$$
\lim \hat{x}(U)=0
$$

for any $x \in L^{1}(T)$; this is the generalised Riemann-Lebesgue lemma. As a corollary, $\hat{x}(U)=0$ for all $U$ save those of a countable subset of $\{U\}$.

We shall begin by applying Theorem 3 to a study of the equation (2) for $x$ and $y$ in a space $L^{p}(T)$ with $1<p<\infty$.

THEOREM 4. Let $\mu$ be a Radon measure on $T$ such that $\|a(U)\|<1$ for all $U \in\{U\}$, let $1<p<\infty$, and let $y$ be a given element of $L^{p}(T)$. Then either 
(a) $\lim _{n}\left\|\mu^{n} * x+y+\mu * y+\cdots+\mu^{n-1} * y\right\|_{L^{p}}=+\infty$ for each $x \in L^{p}(T)$, the powers of $\mu$ being convolution powers; or (b) the equation (2) has a unique solution $x \in L^{p}(T)$.

Proof. Suppose that (a) is false. If we write $F(x)=\mu * x+y$, it follows that there exists $x_{0} \in L^{p}(T)$ such that the sequence $\left(x_{n}\right)=\left(F^{n}\left(x_{0}\right)\right)$ has a bounded subsequence. Let $M$ denote the closed vector subspace of $L^{p}(T)$ generated by all left-translates of $x_{0}$ and of $y$. According to the generalised Riemann-Lebesgue lemma, the transforms $\hat{x}_{0}$ and $\hat{y}$ simultaneously vanish outside some countable subset $\left\{U_{m}\right\}$ of $\{U\} . F$ is a self-map of $M$ since

$$
\mu * x(t)=\int_{T} x\left(s^{-1} t\right) d \mu(s)
$$

is the limit in $L^{p}(T)$ of finite linear combinations of left-translates of $x$. This statement is most easily confirmed (and the minor difficulties relating to a pointwise interpretation of the integral most easily evaded) by regarding the integral as $\int_{T} x_{s} d \mu(s)$, an integral of the continuous vector-valued function $s \rightarrow x_{s}$ from $T$ into $L^{p}(T), x_{s}$ being the class of the function $t \rightarrow x$ $\left(s^{-1} t\right)$.

In applying Theorem 2, supplemented as in $\S 4$, we shall take $p_{m}(x)=$ $\left\|\hat{x}\left(U_{m}\right)\right\|$. For $L$ we choose the closed subspace of $L^{D^{\prime}}(T)=E^{\prime}$ formed of those elements $f$ of $L^{p^{\prime}}(T)$ for which $\hat{f}(U)=0$ for $U$ different from all the $U_{m}$. (As usual, $p^{\prime}$ is defined by $1 / p+1 / p^{\prime}=1$.) The subspace $V$ attached to this choice of the $p_{m}$ is then strongly dense in $L$.

Since there exists a bounded subsequence of $\left(x_{n}\right)$, and since $M$ is closed, this subsequence admits a weak limiting point $x_{\infty} \in M$ (norm-bounded subsets of $L^{p}(T)$ being weakly relatively compact if $\left.1<p<\infty\right)$. This $x_{\infty}$ is a fortiori a limiting point for the topology $\sigma(E, L)$.

Finally, observe that

$$
\widehat{\mu * x(U)}=\hat{\mu}(U) \hat{x}(U) .
$$

We conclude from the supplemented form of Theorem 2 that the equation (2) has a unique solution in $M$. However, it is clear on taking Fourier transforms that (2) can admit at most one solution in $E=L^{D}(T)$.

Remarks. (1) An exactly similar argument applies to the equation

$$
x-x * \mu=y \text {. }
$$

(2) The preceding proof is complicated to the extent of introducing $M$ and $L$, only because at most countably many seminorms $p_{m}$ are admissible. This difficulty evaporates if $T$ is such that $\{U\}$ is countable, as is the case if $T$ is metrisable. In such cases one may take $M=L^{p}(T)$ and use Theorem 3 directly. 
(3) There are two main difficulties standing in the path of dealing likewise with the case $p=1$. In the first place, $L^{1}(T)$ is not reflexive and the Dunford-Pettis criterion for weak relative compactness renders complicated the criterion $\left(A^{\prime}\right)$ in Theorem 2 . In the second place, the finite linear combinations of coordinates of the representations $U$ are no longer strongly dense in the dual space $L^{\infty}(T)$. However, Theorem 2 and the remarks in $\S 4$ permit one to establish an analogue for the space $M(T)$. We regard this as the dual of $X=C(T)$ and recall that the norm-bounded subsets of $M(T)$ are relatively compact for the topology $\sigma(M(T), X)$. The finite linear combinations of coordinates of the representations $U$ are dense in $C(T)$. But one complication remains in comparison with the case $E=L^{p}(T)(1<p<\infty)$ : the transform $\hat{x}$ now no longer vanishes of necessity outside a countable subset of $\{U\}$. With these remarks in mind we may state the following analogue of Theorem 4.

THEOREM 4 bis. Suppose that $T$ is such that $\{U\}$ may be chosen to be countable. The analogue of Theorem 4 remains true if $L^{D}(T)$ be replaced throughout by $M(T)$.

\section{Truncated convolutions over $(0, \infty)$}

There is an analogue of Theorem 4 for the case in which $T$ is taken to be the semi-axis $(0, \infty)$ and the convolutions are truncated. For $s \geqq 0$ and $x \in L^{p}=L^{p}(0, \infty)$ we define $x_{s} \in L^{p}$ by

$$
x_{s}(t)=\left\{\begin{array}{lll}
0 & \text { if } 0<t<s, \\
x(t-s) & \text { if } t>s .
\end{array}\right.
$$

If $\mu$ is any bounded Radon measure on $[0, \infty), \mu * x$ is the element of $L^{p}$ defined by the vector-valued integral

$$
\mu * x=\int x_{s} d \mu(s)
$$

this entails that

for almost all $t$.

$$
\mu * x(t)=\int_{[0, t)} x(t-s) d \mu(s)
$$

For complex $\xi$ with $\operatorname{Re} \xi \geqq 0$ we define

$$
\hat{a}(\xi)=\int e^{-\xi t} d \mu(t)
$$

an analogous definition is made for $\hat{x}(\xi)$ when $x \in I^{p}$ and $\operatorname{Re} \xi>0$. Then

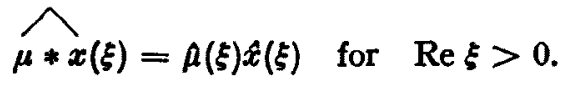

With these notations fixed, the analogue of Theorem 4 runs as follows. 
ThEOREM 5. Suppose that $1<p<\infty$, that $y \in L^{p}=L^{p}(0, \infty)$ is given, and that $\mu$ is a bounded Radon measure on $[0, \infty)$ such that

$$
\text { Inf }\{|\boldsymbol{\mu}(\xi)|: \operatorname{Re} \xi \geqq 0\}<1 .
$$

Then at least one of the following two assertions, (a) and (b), is true:

(a) For each $x \in L^{p}$ it is the case that

$$
\lim _{n}\left\|\mu^{n} * x+y+\mu * y+\cdots+\mu^{n-1} * y\right\|_{L^{p}}=+\infty,
$$

the pozvers of $\mu$ being convolution powers.

(b) The equation

$$
x-\mu * x=y
$$

has a unique solution $x \in L^{p}$.

Proof. We shall apply Theorem 3 with $E=L^{p}$ and $A x=\mu * x$. Assuming (a) to be false, there will exist an $x_{0} \in L^{D}$ such that the sequence $\left(x_{n}\right)$ defined by

$$
x_{n}=A^{n} x_{0}+y+A y+\cdots+A^{n-1} y
$$

has a bounded subsequence. Since $L^{p}$ is reflexive, this bounded subsequence admits a weak limiting point in $L^{p}$. (In fact, since $L^{p^{\prime}}$ is in this case separable, one can extract a weakly convergent subsequence.)

On the other hand, our hypothesis concerning $\mu$ combines with the fact that $A$ is continuous on the half-plane $\operatorname{Re} \xi \geqq 0$ to show that one can find a point $\xi_{0}$ satisfying $\operatorname{Re} \xi_{0}>0$ and a sequence $\left(\xi_{m}\right)$ of distinct points of this open half-plane which converges to $\xi_{0}$ and for which $\left|\hat{\mu}\left(\xi_{m}\right)\right|<1$ for all $m$. Consider the elements $e_{m}^{\prime}$ of the dual space $L^{p^{\prime}}$ defined by $e_{m}^{\prime}(t)=$ $\exp \left(-\xi_{m} t\right)$. The adjoint $A^{\prime}$ of $A$ is easily seen to be defined by

$$
A^{\prime} f=\int_{s} f d \mu(s),
$$

where $f(t)=f(t+s)$. It appears thence that $e_{m}^{\prime}$ is an eigenvector of $A^{\prime}$ belonging to the eigenvalue $\lambda_{m}=\hat{\rho}\left(\xi_{m}\right)$. Accordingly it remains only to show that the finite linear combinations of the $e_{m}^{\prime}$ are dense in $L^{y^{\prime}}$. This well-known result is a consequence of the Hahn-Banach theorem. Thus it suffices to show that if $g \in L^{p}$ has the property that

$$
G(\xi)=\int_{0}^{\infty} g(t) \cdot \exp (-\xi t) d t
$$

vanishes for $\xi=\xi_{m}(m=1,2, \cdots)$, then $g=0$ a.e. Now $G$ is evidently holomorphic for $\operatorname{Re} \xi>0$, and the $\xi_{m}$ have a limiting point $\xi_{0}$ in this domain of holomorphy. The vanishing of $G$ at the points $\xi_{m}$ therefore entails that it vanishes identically on the said half-plane. Equating to zero the successive 
derivatives of $G$ at the point $\xi=1$ gives the equations

$$
\int_{0}^{\infty} g(t) t^{p} \exp (-t) d t=0
$$

for $p=0,1,2, \cdots$. On the basis of Lerch's theorem, one infers that $g=0$ a.e.

Theorem 3 is thus applicable and goes to show that (b) must be true. Thus the negation of (a) implies (b), and Theorem 5 is established.

Remark. If the measure $\mu$ is such that $\mu(\{0\})=0$, then the condition (4) is certainly fulfilled. For in this case when $\xi \rightarrow+\infty$ through real values the functions $e^{-\xi t}$ converge boundedly to zero a.e. for $\mu$, so that correspondingly $\mu(\xi) \rightarrow 0$.

\section{The case of measures and distributions with compact supports}

We conclude with some remarks concerning the application of the method to the equation

$$
x-\mu * x=y
$$

when $\mu$ and $y$ are given, and $x$ is sought, in $M_{0}$ or $D_{0}$. These instances combine some of the features of $\S \S 6$ and 7 .

$M_{c}$ and $D_{c}$ denote, respectively, the spaces of measures and of distributions on $R^{n}$ having compact supports. They are regarded as the duals of $C$ and of $C^{\infty}$, respectively, $C$ being the space of continuous functions with the topology of locally uniform convergence, and $C^{\infty}$ that of indefinitely differentiable functions with the topology of locally uniform convergence of each derivative. $M_{c}$ and $D_{c}$ are endowed with the associated weak topologies.

Three observations form the basis of the application.

(i) $C$ and $C^{\infty}$ are separable Fréchet spaces, so that any bounded sequence in $M_{\mathrm{c}}$ or in $D_{c}$ contains a weakly convergent subsequence, and the strong topologies $\beta\left(C, M_{0}\right)$ and $\beta\left(C^{\infty}, D_{0}\right)$ coincide with the initial topologies on $C$ and $C^{\infty}$ respectively. $\left(C^{\infty}\right.$ is also reflexive and a Montel space, so that weakly convergent sequences in $D_{\mathrm{c}}$ are even strongly convergent.)

(ii) If one arranges as a sequence $\left(\xi_{m}^{0}\right)$ the set of points of the form

$$
\left(r^{-1}, 0, \cdots, 0\right),\left(0, r^{-1}, 0, \cdots, 0\right), \cdots,\left(0, \cdots, 0, r^{-1}\right),
$$

where $r=1,2, \cdots$, the functions $e_{m}^{\prime}(x)=\exp \left(-i \xi_{m} x\right)$, where $\xi_{m}=\xi+\xi_{m}^{0}$ and $\xi$ is an arbitrary fixed point of $Z^{n}$, are total in $C$ and in $C^{\infty} . Z^{n}$ denotes the $n$-dimensional complex number space.

(iii) The Fourier transform

$$
\rho(\xi)=\left\langle e^{-i \xi x}, \mu\right\rangle
$$

is an entire function of $\xi$ so that, unless $\mu=c . \varepsilon(c=$ constant, $\varepsilon=$ Dirac 
measure at 0 ),

$$
\operatorname{Inf}\left\{|\boldsymbol{\mu}(\xi)|: \xi \in Z^{n}\right\}=0 \text {. }
$$

Hence one can find $\xi \in Z^{n}$ such that the $e_{m}^{\prime}$ are total in $C$ and in $C^{\infty}$, and such that $\left|\hat{\mu}\left(\xi_{m}\right)\right|<1$ for all $m$.

One can now apply Theorem 3 directly to conclude that, given $\mu$ and $y$ in $M_{c}$ (or $\left.D_{c}\right), \mu \neq c \cdot \varepsilon$, either for each $x_{0}$ in $M_{c}$ (or $\left.D_{c}\right)$ the sequence $\left(x_{n}\right)$ defined by

$$
x_{n}=\mu^{n} * x_{0}+y+\mu * y+\cdots+\mu^{n-1} * y
$$

contains no subsequence which is bounded in $M_{c}$ (or $D_{c}$ ), or the equation (2) has a unique solution $x$ in $M_{c}$ (or $D_{c}$ ). If $\mu=c \cdot \varepsilon$, equation (2) reduces to the trivial form $(1-c) x=y$.

In the case of $D_{c}$ one has a more direct aid in determining the solubility of (2) in the shape of the Paley-Wiener-Schwartz theorem, which characterises the Fourier transforms of elements of $D_{c}$ as the entire functions of exponential type and of polynomial rate of growth at infinity on $R^{n}$. This may be combined with results concerning the division of functions of this category (see, e.g., [2], p. 38, Théorème 1). Notice that the existence of a bounded subsequence of $\left(x_{n}\right)$ entails that $\hat{y}(\xi)=0$ whenever $\hat{\mu}(\xi)=1$, but this is a priori not sufficient to ensure even that $(1-\hat{\mu})^{-1} \hat{y}$ is an entire function.

Note added in proof. I am grateful to Mr. Fearnley-Sander for the following two interesting remarks: (a) by avoiding direct appeal to Banach's Contraction Principle, Theorem 1 can be proved without assuming either countability or strong boundedness of the family $\left(p_{m}\right)$; (b) Theorem 2 can be established without using condition (iii) in $\S 1$, so that the statement of Theorem 3 and the proofs of Theorems 4 and 5 may be correspondingly simplified.

\section{References}

[1] Edelstein, M., On fixed and periodic points under contractive mappings. Journal London Math. Soc. 37 (1962), $74-79$.

[2] Malgrange, B., Existence et approximation des solutions des équations aux dérivées partielles et des équations de convolution. Thèse. Paris (1955).

Department of Mathematics, Institute of Advanced Studies, A.N.U. 